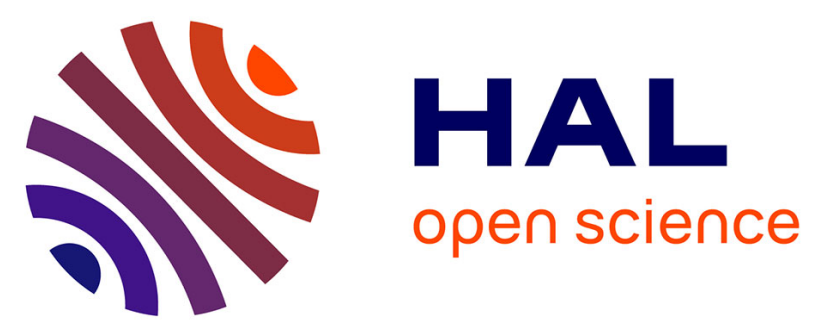

\title{
Autonomous CMOS Power Management Integrated Circuit for Electrostatic Kinetic Energy Harvesters e-KEH
}

\author{
Abdelkrim Bessaad, Amine Rhouni, Philippe Basset, Dimitri Galayko
}

\section{To cite this version:}

Abdelkrim Bessaad, Amine Rhouni, Philippe Basset, Dimitri Galayko. Autonomous CMOS Power Management Integrated Circuit for Electrostatic Kinetic Energy Harvesters e-KEH. 26th IEEE International Conference on Electronics, Circuits and Systems (ICECS 2019), Nov 2019, Genoa, Italy. pp.338-341, 10.1109/ICECS46596.2019.8964736 . hal-02471284

\section{HAL Id: hal-02471284 \\ https://hal.science/hal-02471284}

Submitted on 7 Feb 2020

HAL is a multi-disciplinary open access archive for the deposit and dissemination of scientific research documents, whether they are published or not. The documents may come from teaching and research institutions in France or abroad, or from public or private research centers.
L'archive ouverte pluridisciplinaire HAL, est destinée au dépôt et à la diffusion de documents scientifiques de niveau recherche, publiés ou non, émanant des établissements d'enseignement et de recherche français ou étrangers, des laboratoires publics ou privés. 


\title{
Autonomous CMOS Power Management Integrated Circuit for Electrostatic Kinetic Energy Harvesters e-KEH
}

\author{
Abdelkrim Bessaad ${ }^{1}$, Amine Rhouni ${ }^{1}$, Philippe Basset ${ }^{3}$ and Dimitri Galayko ${ }^{1,2}$ \\ ${ }^{1}$ Sorbonne Université, CNRS, LIP6, F-75005 Paris, France \\ ${ }^{2}$ Yangzhou University, Physics College, Yangzhou, People's Republic of China \\ ${ }^{3}$ Université Paris-Est, ESYCOM, ESIEE Paris, Noisy-le-Grand 93160, France \\ Email : abdelkrim.bessaad@sorbonne-universite.fr
}

\begin{abstract}
In this paper we present an autonomous Power Management Integrated Circuit PMIC for an electret-biased electrostatic kinetic energy harvester with a Bennet's doubler conditioning circuit. The circuit is designed in high voltage 0.35 $\mu \mathrm{m}$ standard bulk CMOS technology. It supplies a low voltage load from the energy extracted from a high voltage transducer. The circuit is provided with a "cold start" mechanism and with a "safe mode" (Recovery) feature that permits to the system to stay active (awake) for a longer time after external vibrations stop temporarily. An ultra-low static power hysteresis comparator is designed for a specific control of the capacitive transducer's conditioning circuit. The autonomy of the system is ensured by a voltage regulator that supplies the internal blocks of the circuit with $1.1 \mathrm{~V}$. The proposed design consumes less than $400 \mathrm{nW}$ making it adequate for use with existing stateof-the art capacitive harvesting MEMS devices.
\end{abstract}

Keywords-Energy harvesting, power management, Bennet's doubler, electrostatic transduction, MEMS, IoT.

\section{INTRODUCTION}

The emergence of the Internet of Things prioritized the power management in the connected devices, so to maximize their autonomy. Many efforts have been made for optimizing the consumed power: introduction of sleep mode, near sensor computing approaches, etc. The autonomy is even more important when access to these devices is difficult or even impossible (space instruments, in-vivo implanted biomedical devices...), and when the battery cannot be replaced easily. Device's autonomy can be ensured by harvesting energy from available ambient sources e.g. light, vibrations, RF waves.

This work proposes an electrical interface implemented in $350 \mathrm{~nm}$ CMOS technology for devices converting vibrational energy into electricity to supply small IoT nodes. The architecture is optimized for electrostatic (capacitive) transducers having a high voltage output interface.

Electrostatic transducers are particularly suitable for miniature electrostatic kinetic energy harvesters (e-KEH) which use MEMS technology. The full architecture of the implemented harvesting system is depicted in Figure 1(a).

Electrostatic transducer is a variable capacitor whose capacitance varies with displacement of its movable electrode. An electret layer is deposited on one of the transducer's electrodes, so providing an initial electrical biasing of transducer and allowing a start-up of the energy harvesting without needing an external energy source. Such transducers are able to provide between 1 and $100 \mu \mathrm{W}$ of power, depending on the particular transducer implementation and on the parameters of external vibrations.
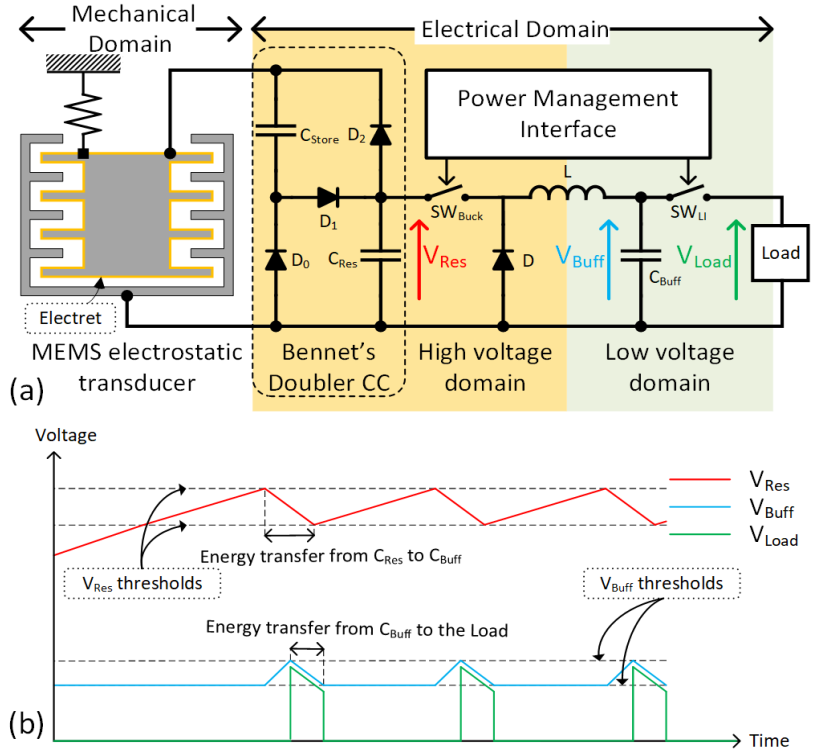

Figure 1 (a) Full e-KEH architecture with the proposed power management interface (b) working principle

A conditioning circuit, based on the Bennet's electricity doubler is used to convert the capacitance variation into electric energy which is available on a reservoir capacitor $\left(\mathrm{C}_{\text {Res }}\right.$ in Figure 1(a)). An additional interface is required in order (i) to transfer the converted energy into a low voltage reservoir capacitor $\mathrm{C}_{\text {Buff }}$ (called buffer in this work), (ii) to manage the transfer rate so to ensure the voltage $V_{\text {Res }}$ to be within the optimal interval. More on the Bennet's doubler operation in the energy harvesting mode can be found in [1]

This work reports on a practical implementation of such an interface called Power Management Integrated Circuit (PMIC). The main technical challenge is that the circuit must operate with an extremely low available power (few microwatts) while managing high voltages. The design was validated by a standard analog IC verification flow and the layout was sent to the foundry for the fabrication.

The proposed design is based on a two-step energy transfer process as shown in Figure 1(b). In the first step, energy is transferred from the conditioning circuit's $C_{\text {Res }}$ to the buffer capacitor $\mathrm{C}_{\text {Buff }}$ through a DC-DC buck converter with an integrated high voltage PMOS switch $\mathrm{SW}_{\text {Buck. }}$ in the second, the energy is transferred from $\mathrm{C}_{\text {Buff }}$ to the load via a PMOS switch $\mathrm{SW}_{\mathrm{LI}}$. The first (second) transfer step begins when a pre-defined upper threshold is detected on $\mathrm{V}_{\text {Res }}\left(\mathrm{V}_{\text {Buff }}\right)$ and it stops when a lower threshold is detected. Notice that the two steps are not synchronized and the control circuits are completely independent from each other. The thresholds of 


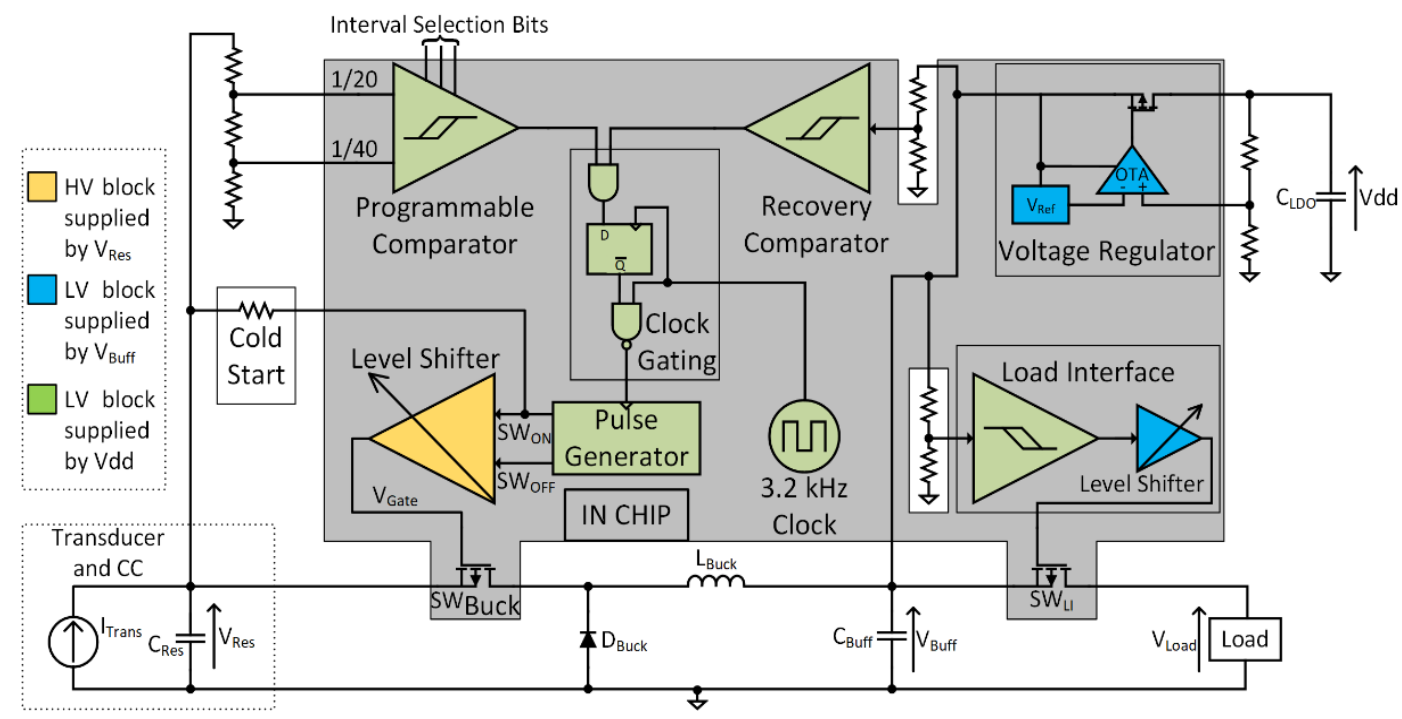

Figure 2 Full e-KEH system showing the detailed architecture of the designed PMIC

$\mathrm{V}_{\text {Res }}$ are supposed to be set-up by an external circuit achieving the MPPT (Maximum Power Point Tracking) function, which is not addressed in this work.

The proposed PMIC is designed to be fully autonomous and to be supplied by a part of the energy generated by the transducer with a reduced voltage of $1.1 \mathrm{~V}$ (compared to the nominal $3.3 \mathrm{~V}$ of the technology), so to ensure an ultra-low power consumption. An original technique is proposed for the the e-KEH's cold start and for "Recovery mode" used to extend the circuit activity time when the external vibrations are temporarily absent or very weak. The proposed design borrows some blocks from the first implementation of the eKEH PMIC published by our team [2]. In the following sections, we describe the main blocks of the PMIC and we present the three operating modes of the e-KEH. Then we present the obtained simulation results.

\section{SYSTEM DESCRIPTION}

The detailed architecture of the designed PMIC within the full e-KEH system is depicted in Figure 2, where the transducer and its conditioning circuit are represented with a DC-current source $\mathrm{I}_{\text {Trans }}$ in parallel with a capacitor $\mathrm{C}_{\mathrm{Res}}=1 \mu \mathrm{F}$. This is a generic model of any capacitive kinetic energy harvester, which always behaves as a charge pump. The main blocks can be split into 3 categories according to their function:

- Voltage regulator: ensures the autonomy of the eKEH by supplying the internal blocks with $1.1 \mathrm{~V}$;

- Load Interface: manages the transfer of energy from the buffer to the load;

- Rest of the blocks: manage the transfer of energy from $\mathrm{C}_{\text {Res }}$ to $\mathrm{C}_{\mathrm{Buff}}$.

\section{A. Working modes}

According to the external vibrations that the e-KEH undergoes, and to the stored energy in its capacitors, there are 3 possible operating modes:

Harvesting mode. In this mode, the e-KEH has enough stored energy in the capacitors and the external vibrations have a sufficient level for the supply power generation. The energy is transferred from $\mathrm{C}_{\mathrm{Res}}$ to $\mathrm{C}_{\mathrm{Buff}}$ via the buck converter according to the multiple energy shot technique. When the programmable comparator detects the selected threshold $\mathrm{V}_{\mathrm{H}}$ on $\mathrm{V}_{\text {Res }}$, it activates the buck converter by allowing the clock signal to pass through the clock gating circuit to the pulse generator. This later, controls the level shifter's output thus turning $\mathrm{ON}$ and $\mathrm{OFF}$ the switch $\mathrm{SW}_{\text {Buck. Once the lower }}$ threshold $\mathrm{V}_{\mathrm{L}}$ is detected on $\mathrm{V}_{\text {Res, }}$, the buck converter is deactivated so that energy re-accumulates in $\mathrm{C}_{\mathrm{Res}}$.

Simultaneously with the buck converter operation, the Load Interface block manages the transfer of energy from the buffer to the load. It is based on the same hysteresis behavior as the buck control circuit. However, in this case, the energy is transferred to the load in one shot through the switch $\mathrm{SW}_{\mathrm{LI}}$. The chosen thresholds are $\mathrm{V}_{\text {Buff- }}=2.25 \mathrm{~V}$ and $\mathrm{V}_{\text {Buff-L }}=2 \mathrm{~V}$.

Cold start. A cold start scenario is a start-up of the e-KEH while the initial stored energy in all of its capacitors is zero. As the used transducer is an electret-biased combined with a Bennet's Doubler conditioning circuit, as soon as external vibrations start, the harvesting process begins and the voltage $\mathrm{V}_{\text {Res }}$ increases [3]. However, without a cold start circuit, the energy would only accumulate in $\mathrm{C}_{\mathrm{Res}}$ and the control blocks would not be supplied. This would lead to the saturation of the $\mathrm{V}_{\text {Res }}$ voltage. The proposed cold start solution consists of a pull-up resistor that forces a positive voltage on the level shifter's $\mathrm{SW}_{\mathrm{ON}}$ input. The e-KEH manages to transfer energy from $\mathrm{C}_{\text {Res }}$ to $\mathrm{C}_{\text {Buff }}$ thereby, supplying the voltage regulator. $\mathrm{V}_{\text {Res }}$ and $\mathrm{V}_{\text {Buff }}$ increase until $\mathrm{V}_{\mathrm{dd}}$ reaches its nominal value and the e-KEH enters to the Harvesting mode.

Recovery. This mode corresponds to the situation where the e-KEH has enough energy stored in the capacitors. However, there are no vibrations. As the internal blocks of the PMIC consume energy, $\mathrm{V}_{\text {Buff }}$ decreases. If the vibrations stop for a long time $\mathrm{V}_{\text {Buff }}$ may reach low values that are insufficient to ensure the correct functioning of the LDO, thus the control blocks. To overcome this situation, we propose to add a hysteresis comparator whose lower threshold $\mathrm{V}_{\text {Rec-L }}$ corresponds to the minimum supply voltage required by the LDO. The Recovery Comparator activates the buck converter when the lower threshold $\mathrm{V}_{\text {Rec-L }}=1.8 \mathrm{~V}$ is detected and it deactivates it when the upper threshold $\mathrm{V}_{\text {Rec-H }}=2 \mathrm{~V}$ is detected. The behavior of the buck converter is the same as explained for the Harvesting mode. The Recovery mode can be seen as a "safe mode", where, the priority is to keep the 


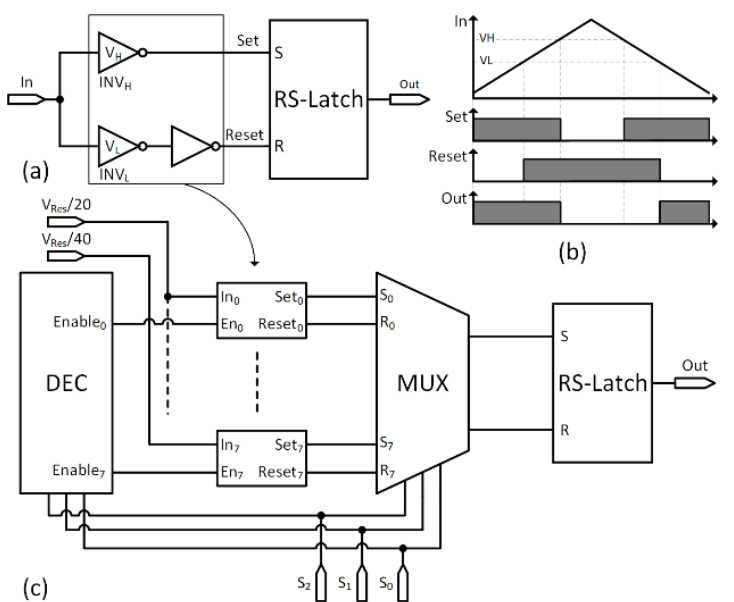

Figure 4 (a) RS-Trigger based hysteresis comparator (b) chronograms (c) programmable hysteresis comparator

system active (awake) as long as possible until the vibrations resume.

\section{B. Circuit description}

This section describes the operation of some key blocks of the PMIC.

Clock generator. This circuit is based on a relaxation oscillator architecture and is inspired by the work reported in [4]. This clock has an ultra-low power consumption of $25 \mathrm{nW}$ and a frequency of $3.2 \mathrm{kHz}$. Implementation details of this circuit, and particularly its adaptation to $1.1 \mathrm{~V}$ supply voltage are described in [2].

Level shifter. It is a dynamic level shifter allowing the gate control of the high-side PMOS switch $\mathrm{SW}_{\text {Buck. Its state is }}$ controlled by short pulses on $\mathrm{SW}_{\mathrm{ON}}$ and $\mathrm{SW}_{\mathrm{OFF}}$ and it has a zero static power dissipation. When it receives a pulse on $S W_{O N}$ its output switches from $V_{\text {Res }}$ to $\left(V_{\text {Res }}-2 \mathrm{~V}\right)$. And when it receives an pulse on $\mathrm{SW}_{\mathrm{OFF}}$, the output switches back to $\mathrm{V}_{\text {Res. }}$. More detail about the level shifter design may be found in[2], [4], [5].

RS-Trigger based hysteresis comparator. This circuit is used in both Recovery Comparator and in Load Interface circuits. It has been previously designed by [2] based on[6], [7]. It is composed of basic logic elements i.e. three inverters and an RS-Latch (see Figure 4(a)). The comparator's thresholds correspond to the inverters $I_{N} V_{H}$ and $I N V_{L}$ thresholds $\mathrm{V}_{\mathrm{H}}$ and $\mathrm{V}_{\mathrm{L}}$, respectively. These thresholds can be fixed by tuning the size ratio W/L of each inverter's transistors. The behavior of the hysteresis comparator is summarized in Figure 4(b) chronogram.

RS-Trigger programmable comparator. To make a programmable hysteresis comparator based on the RS-trigger hysteresis comparator described above, we propose the architecture presented in Figure 4(c). The principle is to duplicate the pair of inverters $\mathrm{INV}_{\mathrm{H}}$ and $\mathrm{INV}_{\mathrm{L}}$ as many times as the number of intervals. Each pair of inverters is then sized according to the required interval. To allow the programmability of the circuit, we use a decoder and a multiplexer. The decoder turns off all unused inverters (in order to reduce power consumption), while the multiplexer selects the outputs of the active pair of inverters and connects its outputs to the RS-Latch's inputs.

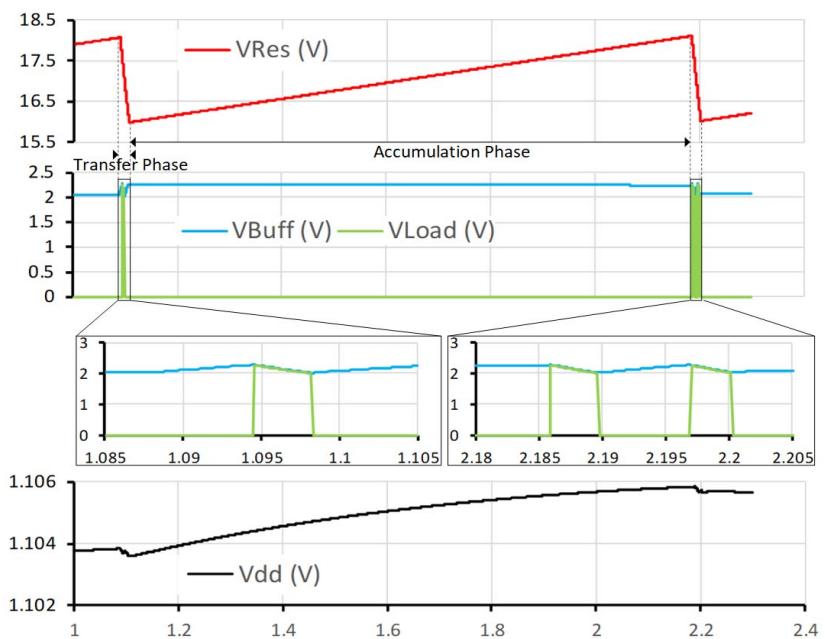

Figure 3 Harvesting mode simulation results

The designed programmable comparator has 3 bits input selection vector, allowing to choose one out of 8 possible intervals of equal length $\mathrm{V}_{\mathrm{H}}-\mathrm{V}_{\mathrm{L}}=2 \mathrm{~V}$. The programmable comparator has two analog inputs, these inputs correspond to the divided (down-scaled) voltages of the output of the conditioning circuit: $\mathrm{V}_{\text {Res }} / 20$ and $\mathrm{V}_{\text {Res }} / 40$. This combination of inputs allows the comparator to cover a wide range of input voltages [8 V $-24 \mathrm{~V}]$.

Voltage regulator LDO. The designed voltage regulator is a Low Drop Out (LDO) voltage regulator that is supplied by $\mathrm{V}_{\text {Buff }}$ and generates a regulated output voltage of $1.1 \mathrm{~V}$. An operational transconductance amplifier OTA is used as an error amplifier in the LDO. The designed reference voltage generator is based on a constant $\mathrm{g}_{\mathrm{m}}$ CMOS quad with an integrated floating resistance of $22.5 \mathrm{MOhm}$. The generated reference voltage is $\mathrm{V}_{\text {Ref }}=672 \mathrm{mV}$. The average quiescent power of the LDO is 42 nW. The minimum supply voltage required for the designed voltage regulator is equal to $1.8 \mathrm{~V}$. Thus, we decided to fix this value as the lower threshold of the Recovery Comparator $\mathrm{V}_{\text {Rec-L }}$ (see sub-sec.A above).

\section{SIMULATION RESULTS}

Several simulations, including MC simulations of individual blocks, have been done to study performance and power consumption of the PMIC. In the following, we present the obtained simulation results of the full e-KEH architecture in all operating modes.

\section{A. Harvesting}

In this simulation, the main performance characteristic to be measured is the efficiency $\eta_{\mathrm{e}-\mathrm{KEH}}$ of the energy transfer from the transducer to the load. The transducer's equivalent current source is fixed to $\mathrm{I}_{\text {Trans }}=2 \mu \mathrm{A}$, and the initial conditions are: $\mathrm{V}_{\mathrm{Res} 0}=16 \mathrm{~V}, \mathrm{~V}_{\mathrm{Buff} 0}=2 \mathrm{~V}$ and $\mathrm{V}_{\mathrm{dd} 0}=1.1 \mathrm{~V}$. The chosen interval for $\mathrm{V}_{\text {Res }}$ is $[16 \mathrm{~V}-18 \mathrm{~V}]$. The average power generated by the transducer model is $34 \mu \mathrm{W}$ : this is within the power range generated by the state-of-the art capacitive kinetic harvesters, which is typically between $10 \mu \mathrm{W}$ and 100 $\mu \mathrm{W}$ [8]. Figure 3 shows the obtained simulation results from 0 to $2.3 \mathrm{~s}$. If we consider the two cycles of accumulation and transfer, we obtain the following results:

- $\quad$ The energy consumed by the load is $\mathrm{W}_{\text {Load }}=48.7 \mu \mathrm{J}$;

- $\quad$ The PMIC consumed $\mathrm{W}_{\text {PMIC }}=7.3 \mu \mathrm{J}$; 


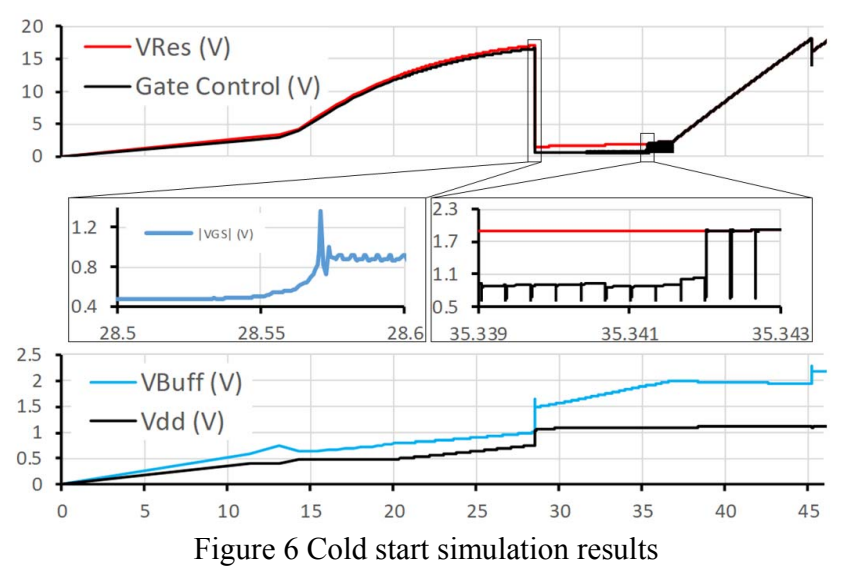

- The energy dissipated by the discrete components is $\mathrm{W}_{\text {other }}=10.8 \mu \mathrm{J}$ of which $\mathrm{W}_{\mathrm{D}-\mathrm{Buck}}=9.8 \mu \mathrm{J}$ is consumed by the flyback diode.

The resulting end to end efficiency is then $\eta_{\mathrm{e}-\mathrm{KEH}}=72.9 \%$.

B. Cold start

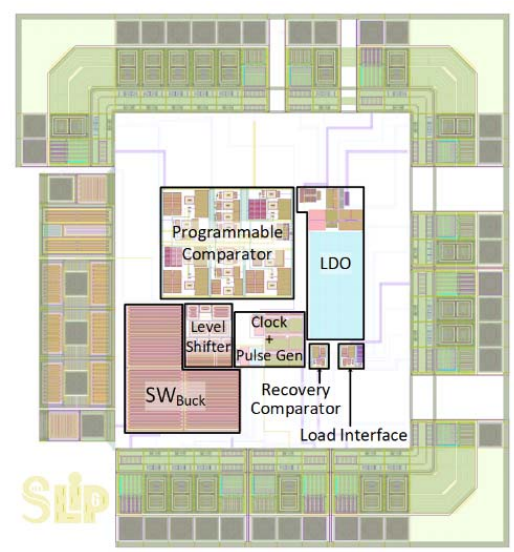

Figure 5 Designed PMIC layout

Initially, $V_{\text {Res }}, V_{\text {Buff }}$ and $V_{d d}$ are null. $I_{\text {Trans }}=2 \mu \mathrm{A}$ during the simulation. The obtained results of a transient analysis are presented in Figure 6.

As $C_{\text {Res }}$ charges from $I_{\text {Trans }}$, a positive voltage is forced by the pull-up resistor at $\mathrm{SW}_{\mathrm{ON}}$. The Level shifter's output is then $V_{\text {Gate }}<V_{\text {Res. }} C_{\text {Res }}$ and $C_{\text {Buff }}$ charge in the same time and $V_{\text {dd }}$ increases. It can be seen at $t=13 \mathrm{~s}$, that $\mathrm{V}_{\text {Buff increases slowly }}$ compared to $\mathrm{V}_{\text {Res. }}$. This is due to the effect of the pulse generator that tries to force $\mathrm{SW}_{\mathrm{ON}}$ to $0 \mathrm{~V}$.

At $\mathrm{t}=28.6 \mathrm{~s}$, the pull-up resistor manages to force a positive voltage on $\mathrm{SW}_{\mathrm{ON}}$ resulting in $\mathrm{V}_{\text {Gate }}<\mathrm{V}_{\mathrm{Th}}=0.63 \mathrm{~V}$ (see zoom from $28.5 \mathrm{~s}$ to $28.6 \mathrm{~s}$ in Figure 6). Energy is then instantly transferred to $\mathrm{C}_{\mathrm{Buff}}$ and $\mathrm{V}_{\mathrm{dd}}$ approaches its nominal value. Here, the buck converter is activated by the Recovery Comparator. In the beginning, the level shifter doesn't manage to operate correctly due to $\mathrm{V}_{\text {Res }}$ low values. until this later reaches a sufficient level (see zoom from $35.339 \mathrm{~s}$ to 35.343 $\mathrm{s})$. At $\mathrm{t}=36.8 \mathrm{~s}, \mathrm{~V}_{\text {Buff }}=\mathrm{V}_{\text {Rec-H}}=2 \mathrm{~V}$ and the buck converter is deactivated. The e-KEH enters to Harvesting mode.

\section{Recovery}

Figure 7 shows the obtained simulation results of the eKEH operating in Recovery mode. The initial conditions are: $\mathrm{V}_{\text {Res0 }}=8 \mathrm{~V}, \mathrm{~V}_{\text {Buffo }}=2.2 \mathrm{~V}, \mathrm{~V}_{\mathrm{dd} 0}=1.1 \mathrm{~V}$. In the beginning $\mathrm{I}_{\text {Trans }}$ $=0 \mathrm{~A}$. Due to internal blocks consumption $\mathrm{V}_{\text {Buff }}$ decreases,
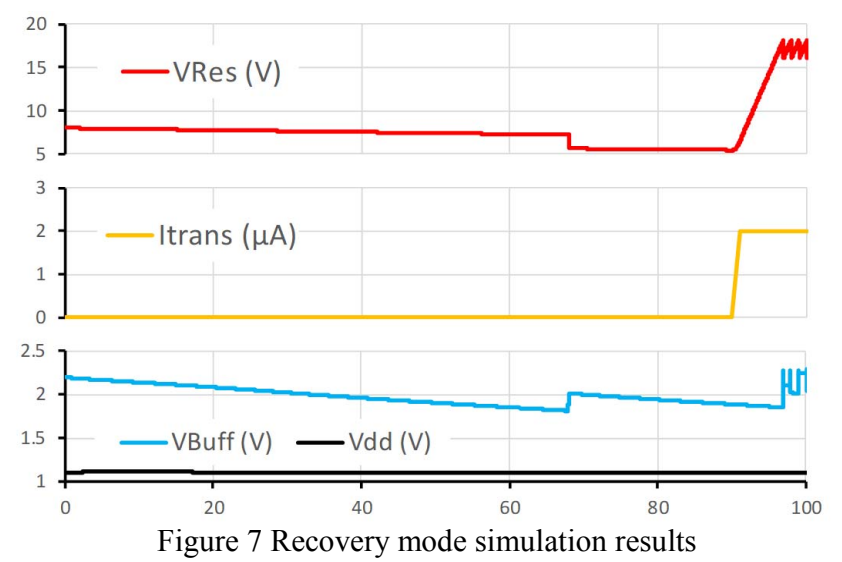

until it reaches the Recovery Comparator's threshold $\mathrm{V}_{\text {Rec- } \mathrm{L}}=$ $1.8 \mathrm{~V}$. The buck converter is then activated and $\mathrm{C}_{\mathrm{Buff}}$ recharges until $\mathrm{V}_{\text {Buff }}=\mathrm{V}_{\text {Rec-H }}=2 \mathrm{~V}$. Note that $\mathrm{V}_{\text {Res }}$ decreases due to the voltage divider and Cold Start's pull-up resistor power dissipation. At $\mathrm{t}=90 \mathrm{~s}$ the transducer's current $\mathrm{I}_{\text {Trans }}=2 \mu \mathrm{A}$, thus $\mathrm{V}_{\text {Res }}$ increases and the e-KEH enters to Harvesting mode.

\section{Implemented chip}

In Figure 5, the full layout of the designed PMIC is depicted. The core occupies an area of $0.7 \mathrm{~mm}^{2}$ from a total area of $4.1 \mathrm{~mm}^{2}$ (pad limited chip). The circuit is primarily designed for input voltages from 8 to $24 \mathrm{~V}$. Though, the technology's maximum rating voltage $50 \mathrm{~V}$ can be reached by adjusting the external components values.

\section{CONCLUSION}

A designed power management circuit is proposed for electrostatic kinetic energy harvesters in a high voltage CMOS technology. It has an ultra-low quiescent power consumption thus, being suitable for available capacitive transducers. The proposed e-KEH architecture is fully autonomous, where the voltage regulator ensures that the internal blocks are supplied from harvested energy. The cold start and the Recovery circuits allow the harvesting process to begin and to be maintained despite the randomness of the external vibrations.

\section{REFERENCES}

[1] V. Dorzhiev, A. Karami, P. Basset, F. Marty, V. Dragunov, and D. Galayko, "Electret-Free Micromachined Silicon Electrostatic Vibration Energy Harvester With the Bennet's Doubler as Conditioning Circuit," IEEE Electron Device Lett., vol. 36, no. 2, pp. 183-185, Feb. 2015.

[2] M. Bedier, "Smart power management silicon integrated interfaces for capacitive vibration energy harvesters," Université Pierre et Marie Curie - Paris VI, 2017.

[3] Y. Lu et al., "A batch-fabricated electret-biased wideband MEMS vibration energy harvester with frequency-up conversion behavior powering a UHF wireless sensor node," J. Micromechanics Microengineering, vol. 26, no. 12, p. 124004, Sep. 2016.

[4] A. Dudka, "Study, optimization and silicon implementation of a smart high-voltage conditioning circuit for electrostatic vibration energy harvesting system," Université Pierre et Marie Curie - Paris VI, 2014.

[5] P. Basset, "Conception, réalisation et test d'un microsystème de transport alimenté à distance," Université de Lille 1, 2003.

[6] V. A. Pedroni, "Low-voltage high-speed Schmitt trigger and compact window comparator," Electron. Lett., vol. 41, no. 22, p. 1213, 2005.

[7] C.-K. Pham, "CMOS Schmitt Trigger Circuit with Controllable Hysteresis Using Logical Threshold Voltage Control Circuit," in 6th IEEE/ACIS International Conference on Computer and Information Science (ICIS 2007), Melbourne, Australia, 2007, pp. 48-53.

[8] H. Toshiyoshi, S. Ju, H. Honma, C.-H. Ji, and H. Fujita, "MEMS vibrational energy harvesters," Sci. Technol. Adv. Mater., vol. 19, no. NRG3, pp. 124-143, May 2018. 